\title{
Numerical study on the influence of initial ambient temperature on the aerodynamic heating in the tube train system
}

\author{
Shijie Bao ${ }^{1,2}$, Xiao Hu², Jukun Wang ${ }^{1,2}$, Tianhao $\mathrm{Ma}^{2}$, Yingyu Rao ${ }^{2}$ and Zigang Deng ${ }^{2^{*}}$ (D)
}

\footnotetext{
* Correspondence: deng@swjtu.cn ${ }^{2}$ State Key Laboratory of Traction Power, Southwest Jiaotong University, Chengdu 610031, China Full list of author information is available at the end of the article
}

\begin{abstract}
The evacuated tube transportation has great potential in the future because of its advantages of energy saving and environmental protection. The train runs in the closed tube at ultra-high speed. The heat quantity generated by aerodynamic heating is not easy to spread to external environment and then accumulates in the tube, inducing the ambient temperature in the tube to rise gradually. In this paper, a three-dimensional geometric model and the Shear Stress Transport (SST) $k-\omega$ turbulence model are used to study the influence of initial ambient temperature on the structure of the flow field in the tube. Simulation results show that when the train runs at transonic speed, the supersonic flow region with low temperature and low-pressure is produced in the wake. The structure of the flow field of the wake will change with the initial ambient temperature. And the higher the initial ambient temperature is, the shorter the low temperature region in the wake will be. The larger temperature difference caused by the low temperature region may increase the temperature stress of the tube and affect the equipment inside the tube. Consequently, the temperature inside the tube can be maintained at a reasonable value to reduce the influence of the low temperature region in the wake on the system.
\end{abstract}

Keywords: Tube train, Initial ambient temperature, Aerodynamic heating, Numerical simulation

\section{Introduction}

With the development of economy and technology of the society, the speed of traveling is demanded further, contributing to looking at a way of transportation at superspeed on the ground, beyond aircraft speed. In the dense atmosphere, the maximum economic speed of high-speed vehicle should not be higher than $400 \mathrm{~km} / \mathrm{h}[1,2,3]$. The measured data from German TR and Japanese Shinkansen show that aerodynamic drag will account for more than $80 \%$ of total drag when the train speed exceeds $400 \mathrm{~km} / \mathrm{h}$ [4]. Besides, the high-speed train will cause serious problems like aerodynamic noise, which increases rapidly with 6 th power to 8 th power of speed [5]. In this background, the tube train transportation $[6,7,8]$ emerges as the times requires. It aims to reduce the aerodynamic drag

(c) The Author(s). 2020 Open Access This article is licensed under a Creative Commons Attribution 4.0 International License, which permits use, sharing, adaptation, distribution and reproduction in any medium or format, as long as you give appropriate credit to the original author(s) and the source, provide a link to the Creative Commons licence, and indicate if changes were made. The images or other third party material in this article are included in the article's Creative Commons licence, unless indicated otherwise in a credit line to the material. If material is not included in the article's Creative Commons licence and your intended use is not permitted by statutory regulation or exceeds the permitted use, you will need to obtain permission directly from the copyright holder. To view a copy of this licence, visit http://creativecommons.org/licenses/by/4.0/. 
of the train under high speed by decreasing air pressure in the tube, so then promote the train to a higher speed. Due to its advantages of environmental protection and energy conservation, it becomes the most potential development of ultra-high-speed rail transit in the future [9]. However, it also brings many new aerodynamic problems. For instance, when the high-speed train runs in a closed tube, owing to tube wall's restrictions, and improper choice of air pressure, it will result in the aerodynamic drag of the train rising sharply. Furthermore, with the increasing speed of the train, some aerodynamic heating problems appear gradually. Heat quantity generated in the tube when the train runs does not radiate out through the surrounding airflow as easily as it does in the open air. Hence, it is much easier to aggregate, which causes higher and higher temperature in the tube. As a result, the strength of the train body and the tube may be affected, which will further endanger the system's safety [10]. Therefore, the aerodynamic problems of the tube trains are worth studying, which can provide basis for the design of tube trains in the future.

In recent years, a growing number of scholars have researched the characteristics related to aerodynamic aspects of the tube train, such as aerodynamic drag, aerodynamic noise, aerodynamic heating, etc. Zhou et al. [11] studied the influence of air pressure and running speed on the aerodynamic drag of trains. It is reported that the higher the speed of the train, the greater the influence of air pressure on the aerodynamic drag of the train. Subsequently, Zhou et al. [12] studied the effect of blockage ratio on aerodynamic drag. It is found that the greater the blockage ratio is, the greater the aerodynamic drag of the train. However, when the blockage ratio is small, the influence of its change on the drag of the train is small, while when the blockage ratio is large, the drag of the train rises sharply. Mi et al. [13] used the dynamic mesh to study the regularities of the influence of train running speed, air pressure and blockage ratio on the aerodynamic drag. The law suggests when the train is running in low air pressure, there is a good linear relationship between the aerodynamic drag and the blockage ratio. And when the air pressure is high, the linear relationship weakens. What's more, the aerodynamic drag has an approximate linear relationship with the square of running speed. Chen et al. [14] analyzed these aerodynamic drag characteristics of different shapes of the head car and the tail car under different air pressures and blockage ratios. The result reflects that the air pressure in the tube of $1000 \mathrm{~Pa}$ and blockage ratio of 0.25 can effectively reduce aerodynamic drag. And under this air pressure, different streamlined shapes of the head car have no significant difference in reducing aerodynamic drag, while the blunt tail can reduce the aerodynamic drag more effectively. Zhang [15] calculated and compared the aerodynamic drag of train with different blockage ratios and different air pressures in the tube, and found that under the same aerodynamic drag condition, the energy saving of vacuum pumping by reducing the cross section is greater than that by increasing the vacuum degree. Ma et al. [16] based on the evacuated tube test device, analyzed that the air pressure, running speed and the blockage ratio are three very important factors leading to the energy loss of the evacuated tube. Liu et al. [17] modeled the aerodynamic calculation model of high-speed train with the evacuated tube under low-pressure environment, studied the influence of air pressure, blockage ratio and train's running speed on the aerodynamic drag of the train, and gave the optimal relationship between air pressure, blockage ratio and train's speed of the evacuated tube transportation system. Bao et al. [18] found the shape of the train body and the structure of the tube can affect the aerodynamic drag, and the blockage ratio is 
the main factor. In addition, Kim et al. [19] also found that when shock waves are generated during the tube train operation, the aerodynamic drag will increase dramatically. It is suggested to keep a low air pressure to alleviate the shock wave effect. Zhou et al. $[20,21]$ further studied the shock wave's structure and its change law of the tube train, and found that the shock wave is mainly composed of expansion wave, reflected shock and normal shock. The intensity of the normal shock wave in front of the head car experiences four states: rapid increase, initial stability, sudden decrease and final stability. Influenced by the interaction between the expansion wave and the reflected shock wave, the wake velocity decreases obviously in the opposite direction of the train's movement. Liu et al. [22] and Zhang et al. [23] conducted studies on the aerodynamic noise of the tube train. The results showed that the greater the running speed of the train, the more the aerodynamic noise will be produced. And when the train runs at a constant speed, the intensity of the aerodynamic noise source of the high-speed train can be reduced by decreasing the air pressure and the blockage ratio.

At present, most researches on the aerodynamic aspects of the tube train are focused on the aerodynamic drag, but the research on aerodynamic heating is less. Jia et al. [24] found that when the running speed of the train and the air pressure in the tube are constant, the aerodynamic heating effect increases exponentially with the increase of the blockage ratio, and with the increase of Mach number, the maximum temperature in the flow field increases in a parabola trend. Duan [25] found that the maximum temperature increases with the decrease of the air pressure in the tube. Dong [26] found that the aerodynamic heating in the tube gradually decreases with the increase of the train running time, which is a monotonous increasing parabola. When the running time of the train is short, the aerodynamic heating increases sharply with the time. With the increase of the running time of the train, the increment of aerodynamic heating decreases gradually until it approaches a certain temperature infinitely. In the further study of aerodynamic heating, Niu et al. [10] and Zhou et al. [27] found that the aerodynamic heating effect will increase significantly when there is a shock wave in the tube. And with the increase of the blockage ratio, the choking limit formed in the flow field will intensify the aerodynamic phenomenon, which will further worsen the aerodynamic heating environment in the tube [28].

The above researches show that when the train runs at ultra-high speed in the tube, the aerodynamic heating effect will make the ambient temperature in the tube increase. If the heat quantity cannot be released to the outside, the heat quantity generated by the next train that passes will further increase the ambient temperature. However, the effect of the initial ambient temperature on the aerodynamic heating effect has not been studied so far. An excessive temperature in the tube may affect some equipment inside the tube, which may endanger the safe running of trains. In addition, in the studies on aerodynamic heating of the tube train, most scholars adopt a two-dimensional geometric model, which makes it impossible to further observe the temperature distribution on the train surface and in the tube. In the future, most of the trains in the tube will be maglev trains [1], some types of the maglev trains' external equipment cannot work normally in the high temperature environment, such as the superconducting maglev of Southwest Jiaotong University [29], whose bottom contains several cryostats similar to the wheels of wheel-rail trains, which need to be far away from high temperature environment. Therefore, it is necessary to study the influence of the initial 
ambient temperature on the aerodynamic heating and the temperature distribution in the tube.

\section{Numerical simulation}

\subsection{Geometric model}

Currently, there is no mature shape of the tube train, so a simplified three-dimensional geometric model of the train was used in this paper. The approximate dimensions of the tube and the train are shown in Fig. 1. The height of the train is $H=3.2 \mathrm{~m}$, which is defined as the flow characteristic length [17]. The total length of the train body is $24.688 \mathrm{H}$, the width of the train is $1.060 \mathrm{H}$, and the nose tip length of the head and tail car is $1.875 H$. The total length of the tube is $125 H$, which consists of a circular arc with a radius of $1.063 \mathrm{H}$ and a plane with a width of $1.407 \mathrm{H}$. The distance from the bottom of the train to that of the tube is about $0.156 \mathrm{H}$, and the distance between the stagnation point of nose tip of the head car and the inlet is $31.250 H$. The blockage ratio $(\beta)$ of this geometric model is about 0.28 . The blockage ratio is defined as the ratio of the maximum cross-section area of the train to the cross-section area of the tube. The stagnation point of the nose tip of the head car is set as the coordinate origin of the flow field.

\subsection{Numerical model}

When the train runs in a closed tube at an ultra-high speed, the flow field around the train is in a turbulent flow state. The turbulence calculation is very important for accurate prediction of aerodynamic heating. In the prediction of aerodynamic heating, the near-wall region is the region where aerodynamic heating produces heat flux exchange, and the viscosity is dominant in the viscous sublayer of the boundary layer [30]. Therefore, a precise prediction of the boundary layer is required. The shear stress transport (SST) $k-\omega$ turbulence model combines the advantages of $k-\varepsilon$ turbulence model with $k-\omega$ turbulence model. It has a good ability to predict the low Reynolds number flow within the boundary layer and the fully developed turbulence flow outside the boundary layer [31]. Consequently, the SST $k-\omega$ turbulence model is used to predict aerodynamic heating in this paper. The speed of the train studied in this paper is 1000 $\mathrm{km} / \mathrm{h}$, and the corresponding Mach number is obviously higher than 0.3 , so the compressibility of air needs to be considered. On the other hand, the tube is sealed, and the

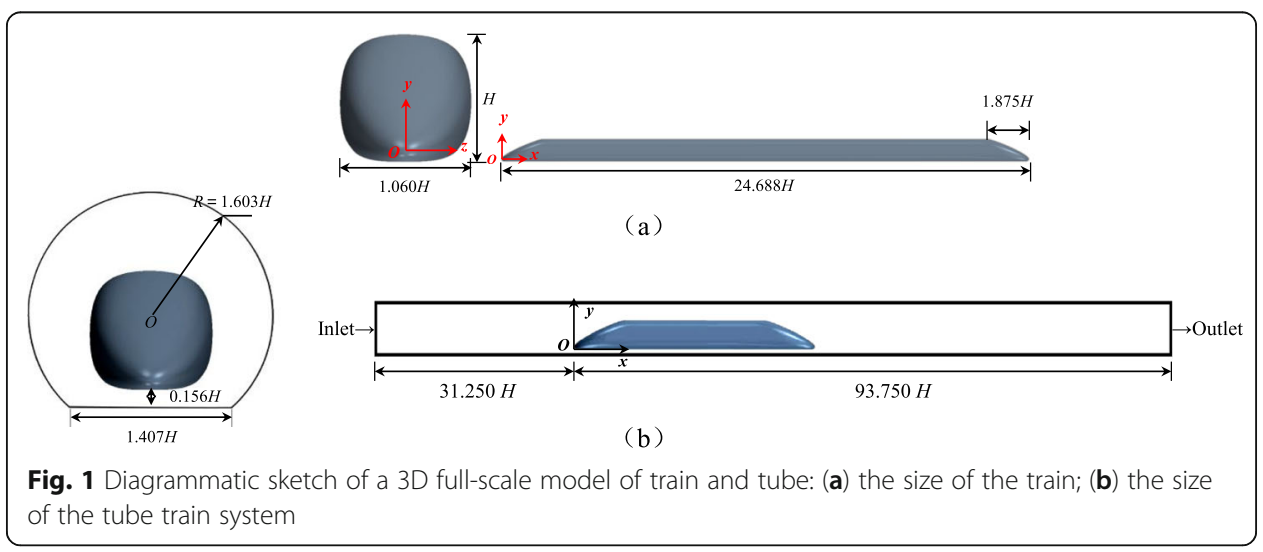


influence of air compressibility also needs to be considered. The flow simulation is based on the finite volume method in STAR-CCM+, and the solver is the coupled implicit steady state solver with the second-order upwind discretization scheme. The AUSM+ scheme can be employed for solving a wide range of flow problems ranging from incompressible to compressible. Hence, the AUSM+scheme was selected to process the inviscid flux to improve the prediction accuracy of aerodynamic heating.

\subsection{Boundary conditions and initial conditions}

As shown in Fig. 2, a diagrammatic sketch of the tube train system is used to describe the boundary conditions of the computational domain. The inlet of the computational domain is defined as the stagnation-inlet, and the outlet is defined as the pressure-outlet. The train is set as the fixed wall with no slip. The thermal boundary conditions of the train and the tube are set as adiabatic. In this paper, we mainly study the aerodynamic heating effect of the tube train at a speed of $1000 \mathrm{~km} / \mathrm{h}$. So, the initial velocity is set to $1000 \mathrm{~km} / \mathrm{h}$. And the reference pressure is $0.1 \mathrm{~atm}(1 \mathrm{~atm}=101,325 \mathrm{~Pa})$ in Section 4. Since this paper studies the influence of initial ambient temperature on aerodynamic heating, the initial temperatures are set at $243 \mathrm{~K}, 293 \mathrm{~K}, 343 \mathrm{~K}$ and $393 \mathrm{~K}$, respectively.

\subsection{Mesh generation}

The trim mesh and the prism mesh in STAR-CCM+ are used to divide the computational domain. The trim mesh is the main volume mesh in the computational domain. For obtaining the flow field information of near-wall area more accurately, the volume mesh of near-wall area of the train surface adopts the prism mesh. The thickness of near-wall prism layer is determined by $y^{+}=1$, about $0.01 \mathrm{~mm}$. The number of prism layers is set to 20 , and the prism layer stretching ratio is set to 1.4. The flow field near the train is complex, especially in the wake of the train. In order to ensure the accuracy of the simulation, the mesh in front of the head car and the mesh in the wake of the tail car are refined with reference to the Muld's [32] mesh layout method. The diagrammatic sketch of the mesh refinement is shown in Fig. 3. The minimum size of the trim mesh is defined as $L_{\mathrm{min}}$. The mesh size of the front of the head car is $L_{\mathrm{min}}$. Due to the wake region of the train being longer, 3 mesh refinement blocks are arranged at the rear of the train, and the size is gradually increased from $L_{\min }$ to $4 L_{\mathrm{min}}$. Figure 4 shows the longitudinal section of the mesh near the train.

\section{Verification}

\subsection{The influence of fixed wall on calculation}

In the computational domain, the tube wall is very close to the train, so setting the tube wall to a fixed wall may affect the calculation results. It is necessary to make a simple discussion on whether the fixed wall has an influence on the calculation results. In this

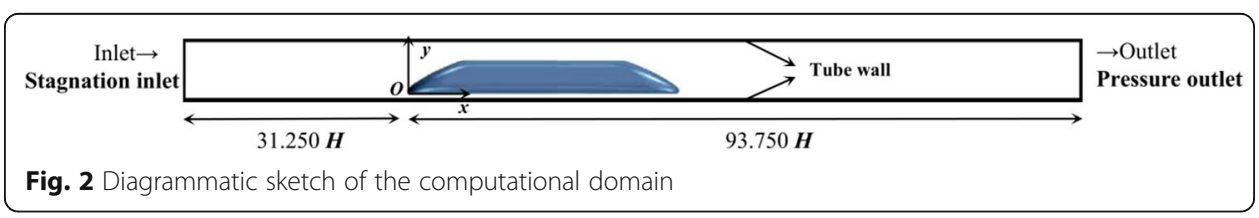




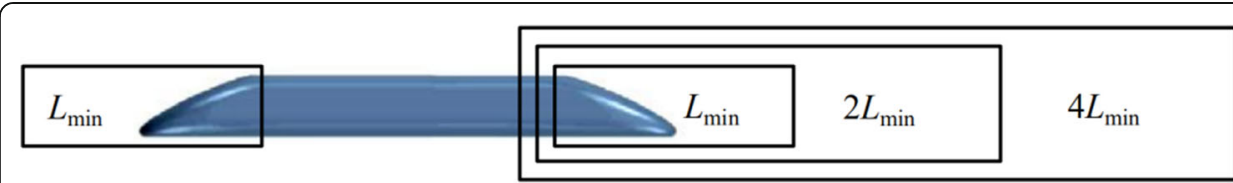

Fig. 3 Diagrammatic sketch of the volume mesh refinement near the train

part, the boundary condition of the tube wall is set as the moving wall with no slip and the fixed wall with no slip respectively. The air pressure in the tube is $0.5 \mathrm{~atm}$. The tangential velocity of the moving wall is equal to the airflow velocity. The initial ambient temperature in the tube is $293 \mathrm{~K}$. The number of mesh is about 18.8 million, and the $L_{\text {min }}=0.05 \mathrm{~m}$ (approximately $0.0156 H$ ).

In this section, the drag coefficient $\left(C_{\mathrm{d}}\right)$ and pressure coefficient $\left(C_{\mathrm{P}}\right)$ are defined as follows:

$$
\begin{aligned}
C_{\mathrm{d}} & =\frac{2 F_{\mathrm{d}}}{\rho_{0} v^{2} S_{\text {train }}} \\
C_{\mathrm{P}} & =\frac{2 P}{\rho_{0} v^{2}}
\end{aligned}
$$

where $F_{\mathrm{d}}$ and $P$ are the aerodynamic drag and the pressure measured in the flow field, respectively. And the pressure $(P)$ in this paper represents the difference between absolute pressure and reference pressure. $\rho_{0}$ is the initial density of air in the flow field. When the air pressure is $0.5 \mathrm{~atm}$ and the ambient temperature is $293 \mathrm{~K}$, the air density is about $0.602 \mathrm{~kg} / \mathrm{m}^{3} . v$ is the train speed $(1000 \mathrm{~km} / \mathrm{h})$, and $S_{\text {train }}$ is the maximum crosssection area of the train, which is about $9.637 \mathrm{~m}^{2}$ here.

Table 1 shows the aerodynamic drag coefficients of the train calculated by using a moving wall and a fixed wall, respectively. The difference of aerodynamic drag coefficient $\left(C_{\mathrm{d}}\right)$ between the moving wall and the fixed wall is great.

Figure 5 shows the distribution of temperature and $C_{\mathrm{P}}$ on the intersection line between the train upper surface and the $x y$ plane, respectively. Here, the surface above the train stagnation point is defined as the upper surface, and the surface below the train stagnation point is the lower surface. It can be seen from Fig. 5 that when the boundary condition of the tube wall is the fixed wall, the distribution of temperature and $C_{\mathrm{P}}$ is quite different from that of the moving wall. The reason for this difference should be that the distance from the tube wall to the train is very short, and the boundary conditions have great interference in solving the flow field around the train.

Therefore, when the wind tunnel model is adopted to calculate the flow field of the tube train, it is more reasonable to set the boundary condition of the tube to the

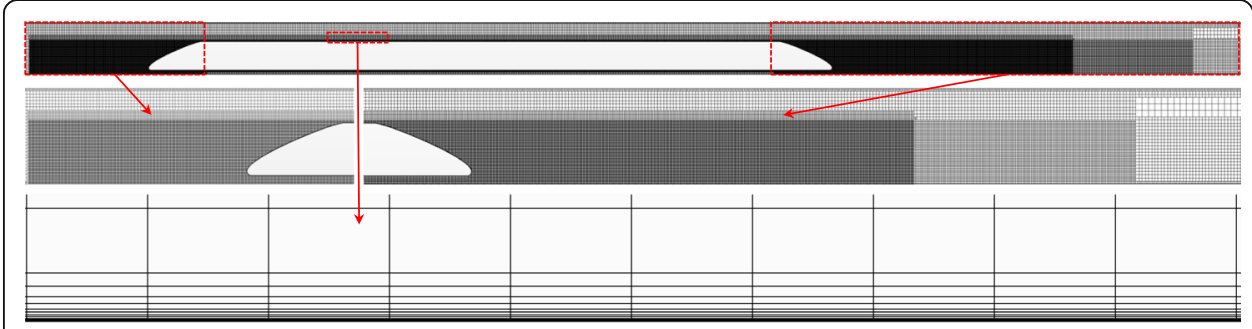

Fig. 4 Longitudinal section of the volume mesh near the train 
Table 1 Drag coefficient

\begin{tabular}{lll}
\hline Item & Moving wall & Fixed wall \\
\hline$C_{d}$ & 2.2776 & 0.4743 \\
\hline
\end{tabular}

moving wall, so that the train moves relative to the tube, which is more consistent with the actual situation. Based on this result, in the following calculations, the tube wall is all set as the moving wall.

\subsection{The influence of prism layer stretching ratio on calculation}

In this paper, the first prism layer is a little thin, about $0.01 \mathrm{~mm}$. In order to ensure a good transition from prism mesh to trim mesh, and avoid the excessive prism layers resulting in a large number of meshes, the stretching ratio of prism layer is set to 1.4. In this section, a brief comparison will be made between the calculation results of the stretching ratio 1.4 and 1.2, so as to explain the rationality of setting the stretching ratio to 1.4. The initial conditions in this section are the same as in Section 3.1.

Table 2 shows the train drag coefficient calculated from different stretching ratios. The drag coefficient is 2.2776 calculated by stretching ratio 1.4 , and the error is only $0.33 \%$ compared with the drag coefficient 2.2701 calculated by stretching ratio 1.2 .

Figure 6 shows the temperature and pressure coefficient distribution on the train surface at different stretching ratios. The temperature of stretching ratio 1.2 is slightly higher than that of stretching ratio 1.4, and the temperature difference at the same position of train surface is less than $1 \mathrm{~K}$. In addition, the distribution of pressure coefficient on the train surface at different stretching ratios is almost the same.

According to the above, it is reasonable to set the prism layer stretching ratio at 1.4. Moreover, the total number of mesh is approximately $32 \%$ higher at stretching ratio 1.2 than at 1.4. Therefore, taking into account the limited computing resources, the prism layer stretching ratio in this paper is set to 1.4.

\subsection{Mesh independence verification}

In order to ensure the rationality of the calculation, three different sizes of the mesh were generated to observe the influence of the number of mesh on the calculation results. Table 3 shows the details of the mesh, including the minimum size of the trim
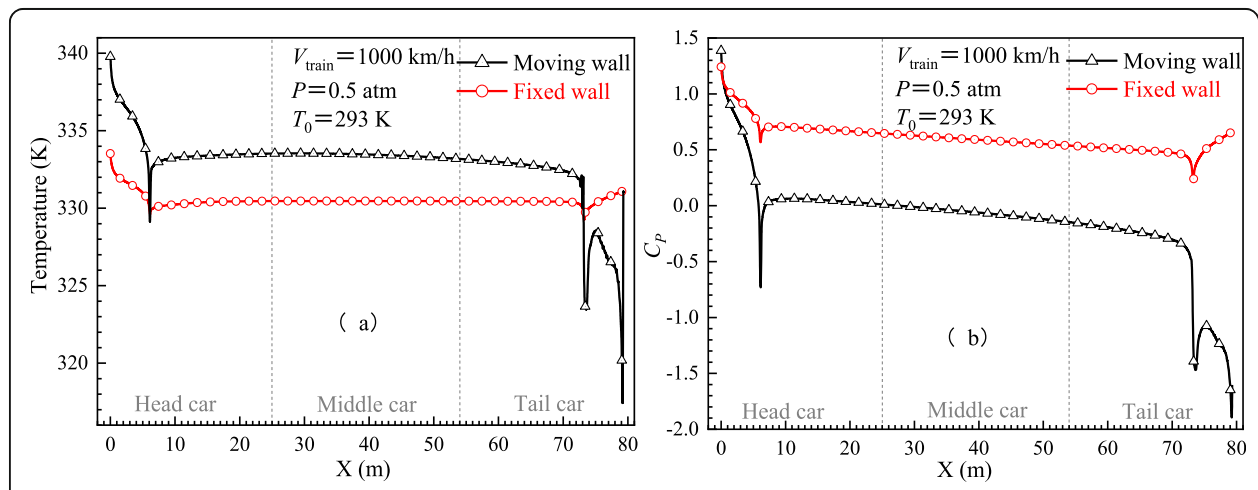

Fig. 5 Temperature and pressure coefficient $\left(C_{p}\right)$ distribution on the intersection line between the train upper surface and the $x y$ plane: a Temperature distribution; $\mathbf{b} C_{p}$ distribution 
Table 2 Drag coefficient calculated from different stretching ratios

\begin{tabular}{lll}
\hline Items & Stretching ratio $\mathbf{1 . 4}$ & Stretching ratio $\mathbf{1 . 2}$ \\
\hline$C_{d}$ & 2.2776 & 2.2701 \\
\hline
\end{tabular}

mesh $L_{\min }$ and the total number of the mesh. The initial conditions in this section are the same as in Section 3.1.

Table 4 shows the $C_{\mathrm{d}}$ calculated from the coarse, medium and fine mesh, respectively. The difference of the $C_{\mathrm{d}}$ between coarse mesh and fine mesh is $0.33 \%$; the $C_{\mathrm{d}}$ of the medium mesh is closer to that of the fine mesh, and the difference is only $0.05 \%$.

Figure 7 shows the distribution of temperature and $C_{\mathrm{P}}$ on the intersection line between the train upper surface and the $x y$ plane under the three types of the mesh. It can be found that both the temperature and $C_{\mathrm{P}}$ of coarse and medium meshes are comparable to those of fine mesh, and the temperature and $C_{P}$ of medium mesh are closer to that of fine mesh. In addition, the temperature and $C_{\mathrm{P}}$ curves of the coarse mesh fluctuate at the shoulder of the tail car, while those of the medium and the fine mesh are relatively gentler. Overall, the calculation results of medium and fine mesh are more reasonable. Considering that the fine mesh will consume more time and computing resource, it is a better choice to use medium mesh to achieve reasonable calculation results.

\subsection{Numerical method verification}

In this section, the ONERA M6 wing, a CFD validation case, is used to verify the numerical method adopted in this paper. The numerical method is adopted to calculate the pressure coefficient of airfoil surface, and the pressure coefficient results are compared with the experimental values of Schmitt and Charpin [33]. The numerical simulations use the flow field conditions of the ONERA M6 wing in Reference [33], as shown in Table 5.

Figure 8 shows the pressure coefficient at several wing span locations, and the numerical results of the pressure coefficient were compared to the experimental values. It can be seen from Fig. 8 that the numerical results of the pressure coefficient on each section of the wing are approximately consistent with the experimental results. Consequently, the numerical method adopted in this paper is reasonable.
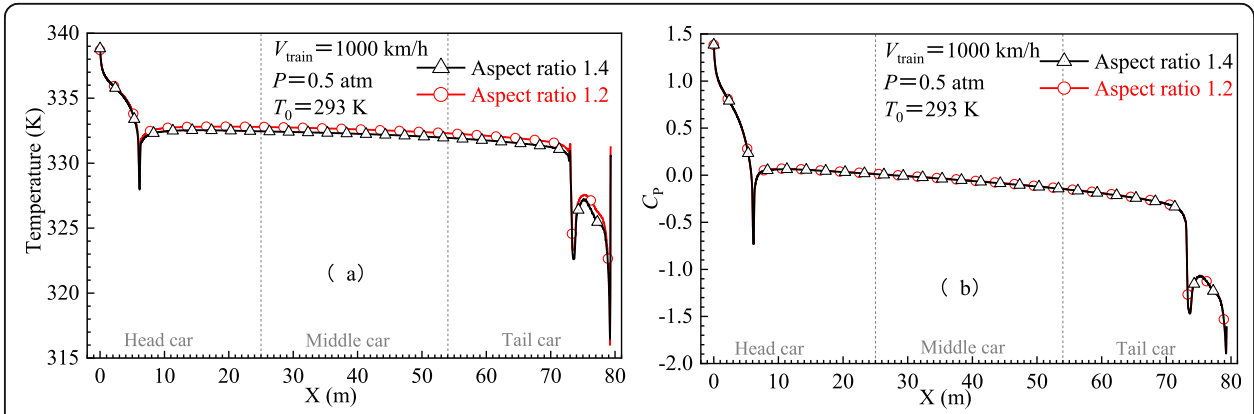

Fig. 6 Temperature $(\mathbf{a})$ and $C_{p}(\mathbf{b})$ distribution on the intersection line between the train upper surface and the $x y$ plane at different stretching ratios 
Table 3 Details of the three types of the mesh

\begin{tabular}{llll}
\hline Items & Coarse mesh & Medium mesh & Fine mesh \\
\hline$L_{\text {min }}$ & $0.0188 \mathrm{H}$ & $0.0156 \mathrm{H}$ & $0.0125 \mathrm{H}$ \\
Total number of mesh (million) & 10.4 & 18.8 & 29.1 \\
\hline
\end{tabular}

\section{Results and discussion}

\subsection{Temperature distribution}

To observe the temperature distribution in the tube, the initial ambient temperature of $293 \mathrm{~K}$ and the air pressure of $0.1 \mathrm{~atm}$ was taken as an example. Figure 9 shows the temperature distribution on the train surface. Line A and line B represent the intersection lines between the upper and lower surface of the train and the $x y$ plane, respectively. Line $C$ represents the intersection line between the left surface of the train and the $x z$ plane. As it can be seen from Fig. 9, the temperature of the train surface is between $308 \mathrm{~K}$ and $340 \mathrm{~K}$, which is higher than the initial ambient temperature. The highest temperature is located at the stagnation point of the nose tip of the head car, near $340 \mathrm{~K}$, and the lowest temperature is located at the nose tip of the tail car, about $315 \mathrm{~K}$. In addition, the temperature distribution of the nose tip of the head and tail car shows a large gradient. The temperature distribution on the middle part of the train body is relatively flat, and the temperature gradient is small.

Figure 10 shows the temperature distribution on the tube wall. Along the tube wall, temperature data on six lines were collected, and the arrangement of these six lines is shown in the figure. With the operation of the train, under the condition that the flow field is stable, it can be found that the temperature difference on the cross section of the same tube wall in front of the wake is small. Because of the complex wake area, the temperature distribution on the tube wall which is located behind the train fluctuates with different amplitude. And there is a low temperature region in the wake area, which makes the temperature of the tube wall also very low. Behind the low temperature region, some locations on the tube wall reach higher temperatures. Subsequently, the temperature distribution on the tube wall tends to be consistent.

Figure 11 shows the change of Mach number along the gap between the train and the tube. The air flowing into the gap between the train and the tube is similar to the air flowing into the Laval nozzle. The nose tip of the head car together with the tube wall forms a convergent section, and the nose tip of the tail car together with the tube wall forms a divergent section. As shown in Fig. 11, the air flows into the gap from the nose tip of the head car at subsonic speed and accelerates continuously in the gap, then the velocity exceeds the local sound velocity at the shoulder of the tail car. Because the transonic fluid no longer follows the principle of "The smaller the cross section, the faster the airflow velocity; the larger the cross section, the slower the airflow velocity". On the contrary, the larger the cross section, the faster the velocity. Therefore, the airflow continuously accelerates and expands, creating expansion waves and compression waves behind the train. As a result, a supersonic flow and low-pressure region are

Table 4 Drag coefficients $\left(C_{d}\right)$ for different meshes

\begin{tabular}{lllll}
\hline Items & Coarse mesh & Medium mesh & Fine mesh & Error (relative to fine mesh) \\
\hline$C_{d}$ & 2.2689 & 2.2776 & 2.2764 & $0.33 \%$ and $0.05 \%$ \\
\hline
\end{tabular}




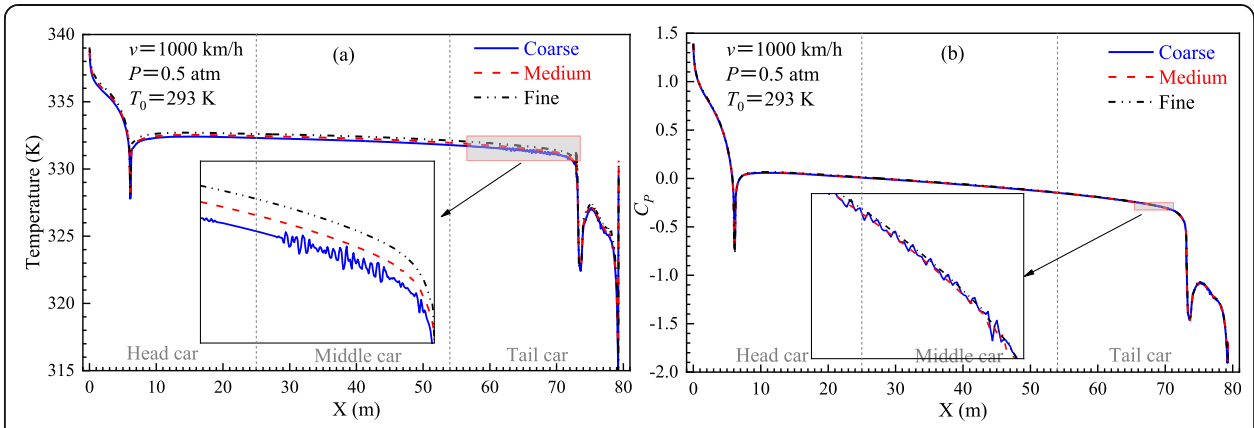

Fig. 7 Temperature $(\mathbf{a})$ and $C_{p}(\mathbf{b})$ distribution on the intersection line between the train upper surface and the $x y$ plane

formed in the wake, shown in Fig. 12. In addition, because the tube wall and the train surface are adiabatic, the flow field in the tube can be regarded as isentropic flow. Eventually, the temperature in the supersonic flow region becomes very low, forming a low temperature region.

When the airflow velocity in the flow field reaches the local sound velocity, it is possible to generate shock waves. Figure 12 shows the contours of pressure, temperature and Mach number. As analyzed earlier, the Mach number of the train's tail exceeds 1 and the maximum Mach number reaches 2. Therefore, shock waves are generated at the rear of the train. Figure 12(b) shows the horizontal cross-section of the contour of pressure, temperature and Mach number in the wake area, respectively. It can be seen from the figure that the expansion wave has an obvious influence on the structure of the flow field in the wake area. Due to the limitation of the tube wall, the continuous reflection and interaction of the compression wave significantly change the structure of the flow field in the tube and complicate the wake.

\subsection{The influence of initial ambient temperature $\left(T_{0}\right)$ on flow field}

In the future, several high-speed trains will continuously run in the tube, so the ambient temperature inside the tube will be constantly rising. For the trains running in the tube at different times, the initial ambient temperature $\left(T_{0}\right)$ of the flow field is different. The earlier the train runs, the lower the ambient temperature, and the later the train runs, the higher the ambient temperature may be. Accordingly, this section mainly discusses the influence of $T_{0}$ on the structure of flow field. $T_{0}$ assumed in the calculation cases in this section are $243 \mathrm{~K}, 293 \mathrm{~K}, 343 \mathrm{~K}$ and $393 \mathrm{~K}$, respectively. The assumptions for some of $T_{0}$ may not be in line with the actual situation, which is only for exploratory discussion here.

Figure 13 shows the pressure distribution on the intersection line between the train upper surface and the $x y$ plane. It can be seen from Fig. 13 that pressure distribution under different $T_{0}$ is roughly approximate as a whole. As $T_{0}$ rises, the pressure on the train surface decreases gradually. At the nose tip of the tail car, the pressure

Table 5 Flow conditions of the ONERA M6 wing in Ref. [33]

\begin{tabular}{llll}
\hline Mach & Reynolds number & Attack angle & Sideslip angle \\
\hline 0.8395 & $11.72 \times 10^{6}$ & $3.06^{\circ}$ & $0^{\circ}$ \\
\hline
\end{tabular}




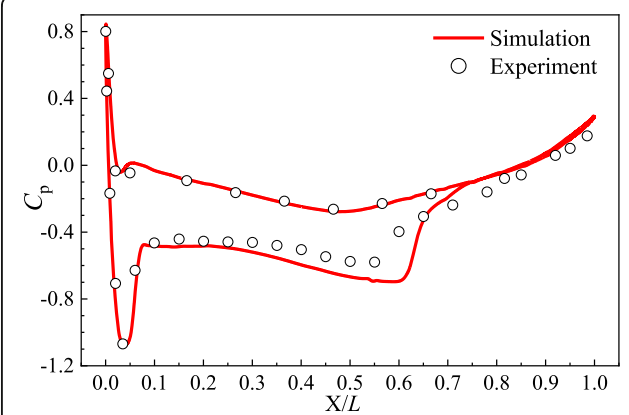

(a) $y / b=0.2$

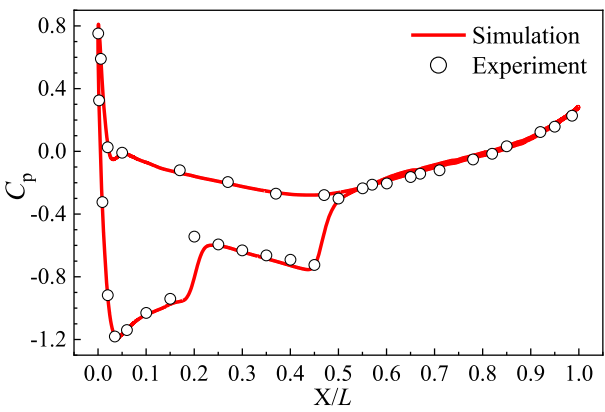

(c) $y / b=0.65$

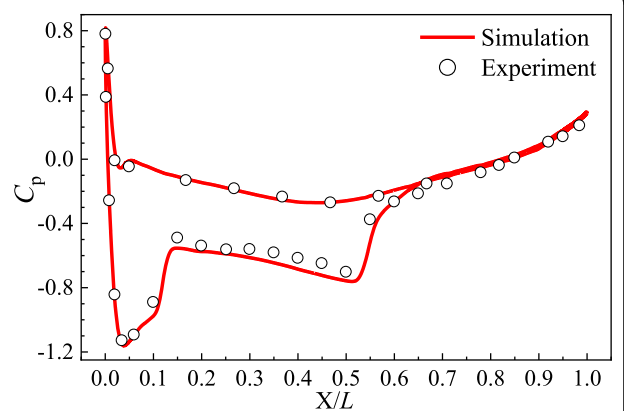

(b) $y / b=0.44$

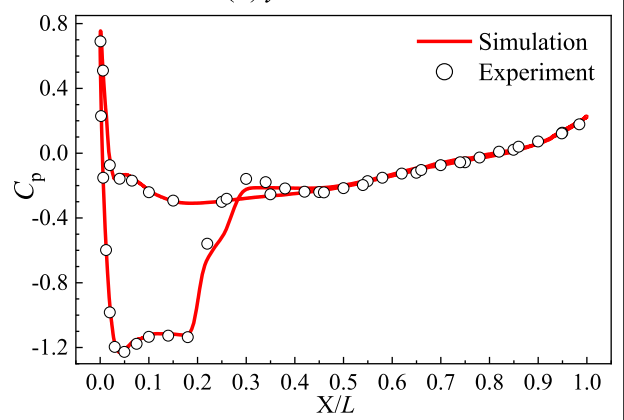

(d) $y / b=0.95$

Fig. 8 Pressure coefficients at several wing span locations of the ONERA M6 wing

distribution at $T_{0}=243 \mathrm{~K}$ is similar to that at $T_{0}=293 \mathrm{~K}$. When the $T_{0}$ is $343 \mathrm{~K}$ and $393 \mathrm{~K}$, the pressure distribution at the nose tip is quite different from that of $T_{0}=243 \mathrm{~K}$ and $293 \mathrm{~K}$. In addition, the maximum pressure on the train surface gradually decreases, and the minimum pressure gradually rises, as shown in Fig. 14. The pressure change on the train surface further affects the aerodynamic drag. Figure 15 shows the

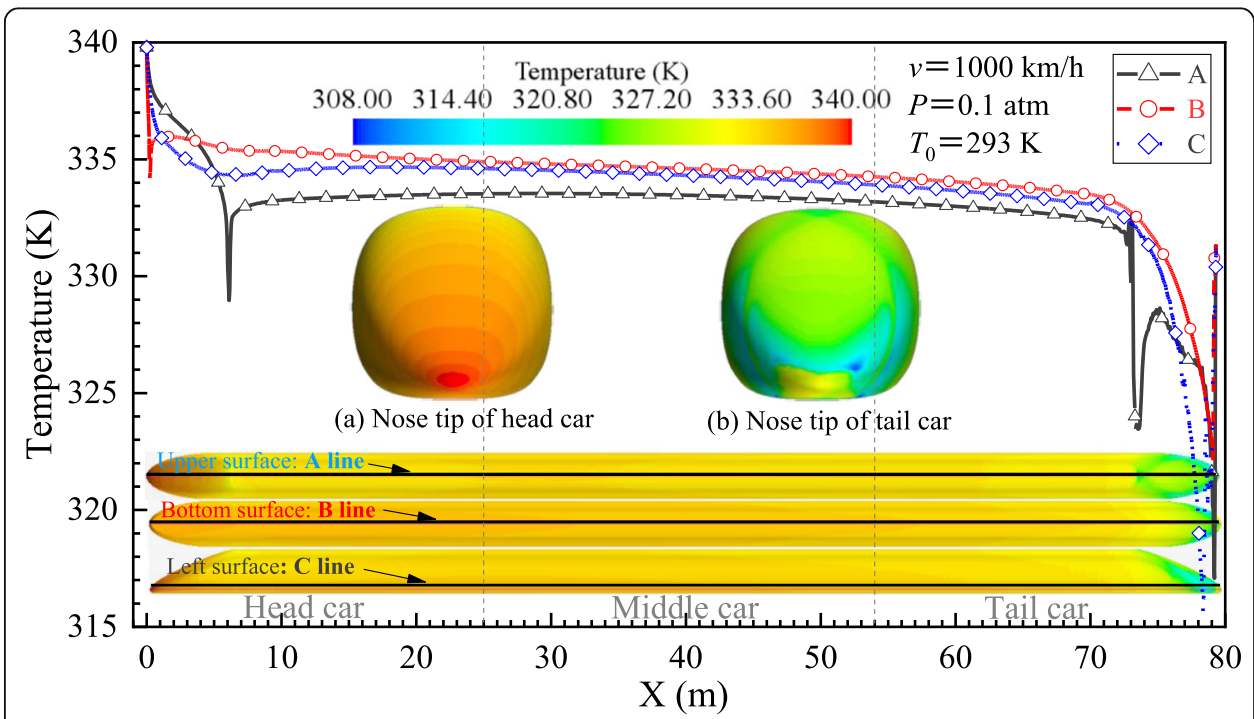

Fig. 9 Temperature distribution on train surface: line A and line B are the intersection lines between the upper and lower surface of the train and the $x y$ plane, respectively; line $C$ is the intersection line between the left surface of the train and the $x z$ plane 


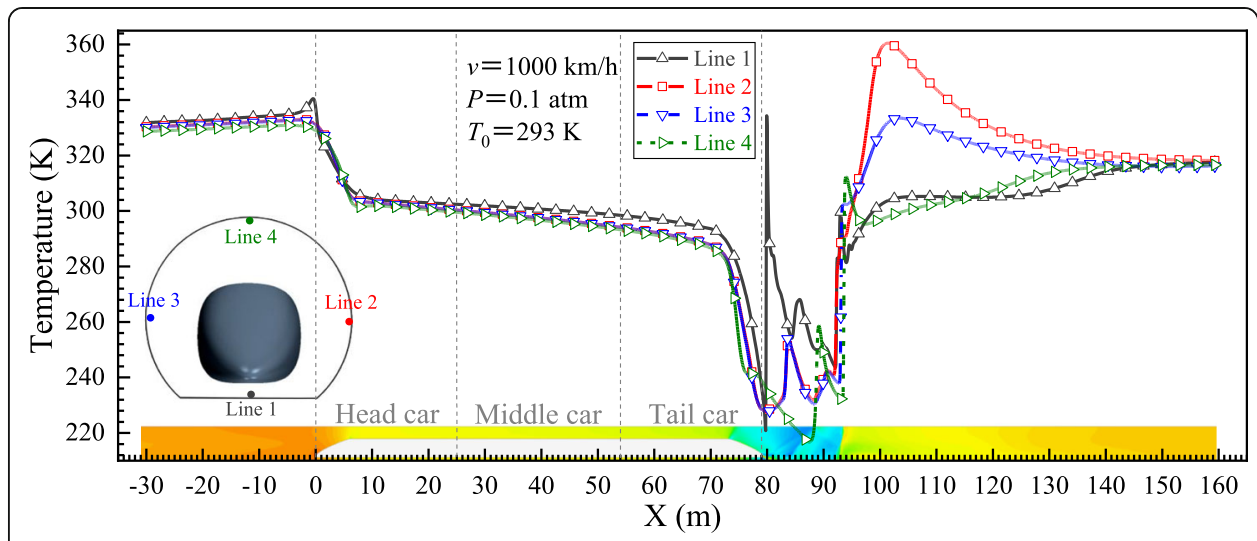

Fig. 10 Temperature distribution along the tube wall: $x$-coordinate ranges from $-30 \mathrm{~m}$ to $160 \mathrm{~m}$

aerodynamic drag of the train at different $T_{0}$. As shown in the figure, the aerodynamic drag decreases with the increase of $T_{0}$. And the aerodynamic drag of the train at $T_{0}=$ $393 \mathrm{~K}$ is about $50 \mathrm{kN}$ lower than that of $T_{0}=243 \mathrm{~K}$. The aerodynamic drag consists of pressure drag and shear force. The pressure drag accounts for $90 \%$ of the aerodynamic drag, so the pressure drag plays a leading role and is the main reason for the decrease of the aerodynamic drag. Therefore, the decrease of pressure drag is the main factor leading to the decrease of aerodynamic drag.

To explore the influence of $T_{0}$ on the Mach number, the Mach number on the straight line near the top of the tube was collected, as shown in Fig. 16. The $x$-coordinate of the straight line ranges from -10 to $110 \mathrm{~m}$, and the $x$-coordinate of the nose tip of the tail car is about $80 \mathrm{~m}$, so this is also the position at the beginning of the wake area. It can be seen that the Mach number distribution in front of the wake area is basically consistent at different $T_{0}$. However, the distribution of Mach number in the wake area is quite different. Combined with the contour of the Mach number (as shown in Fig. 17) in the wake area, it can be found that $T_{0}$ has a great influence on the supersonic flow region. When $T_{0}$ is low, there is a longer supersonic flow region appearing in the wake area, and when $T_{0}$ increases, the supersonic flow region becomes

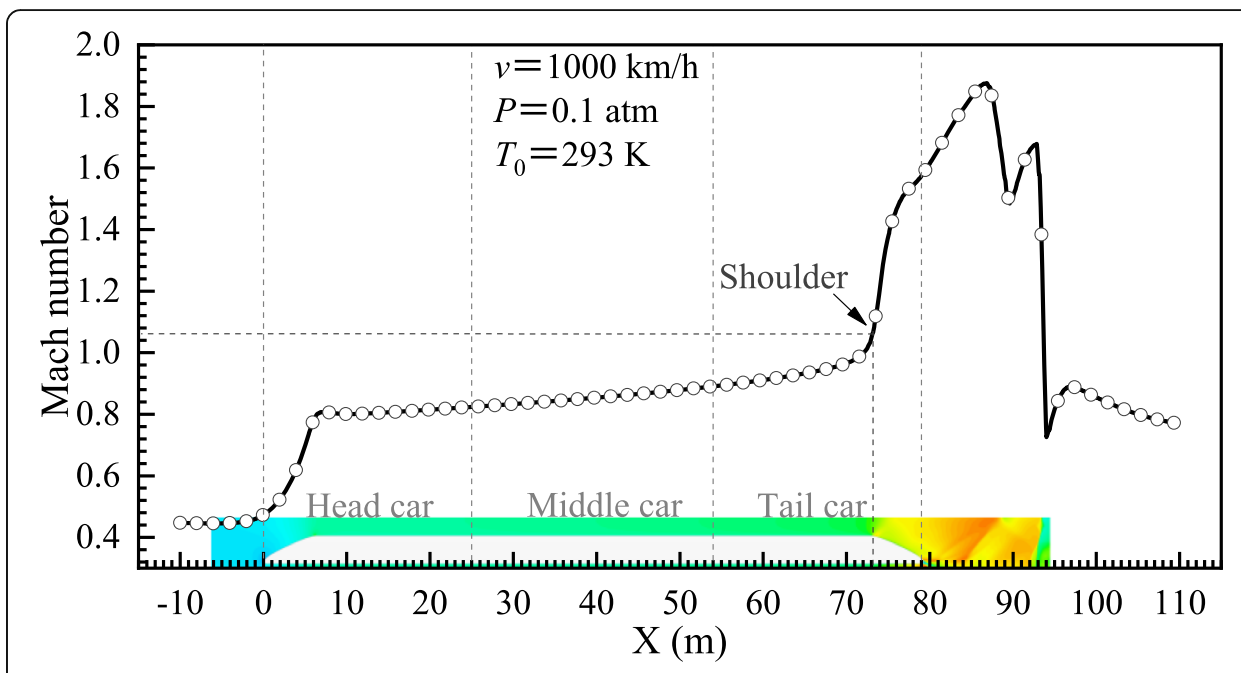

Fig. 11 Mach number along the top of tube wall: $x$-coordinate ranges from $-10 \mathrm{~m}$ to $110 \mathrm{~m}$ 


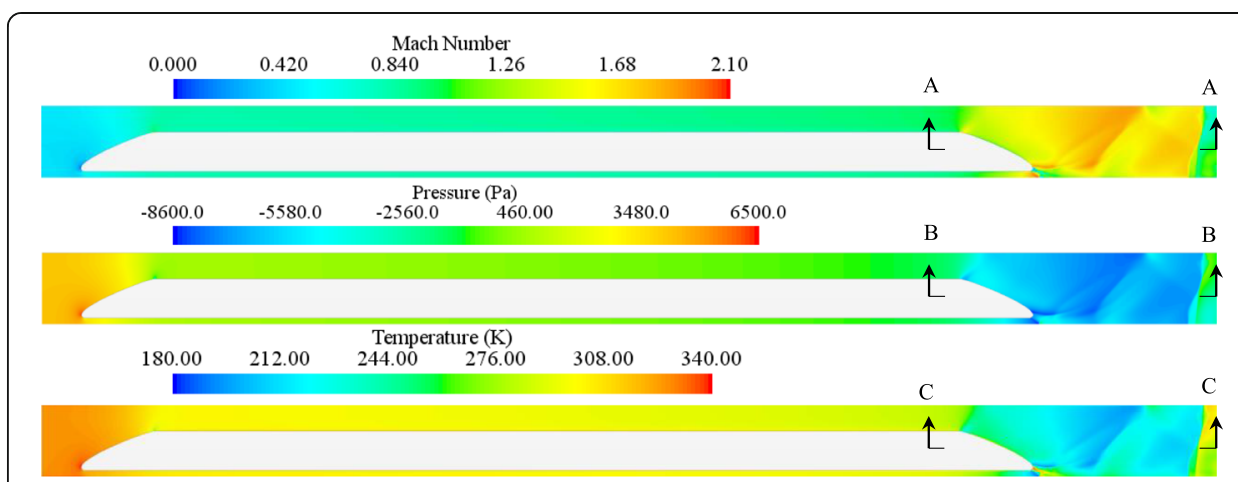

(a)

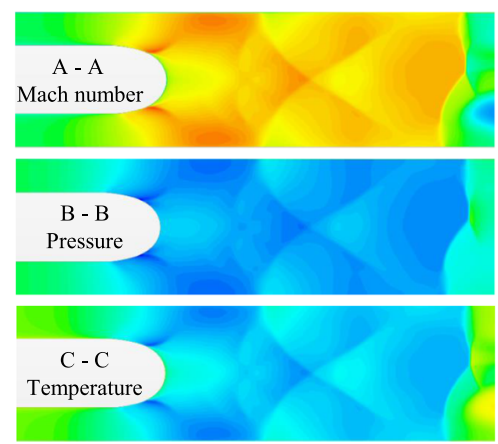

(b)

Fig. 12 The contours of pressure coefficient, temperature and Mach number: a The longitudinal section of the flow field around the train; $\mathbf{b}$ The horizontal cross-section of the wake area

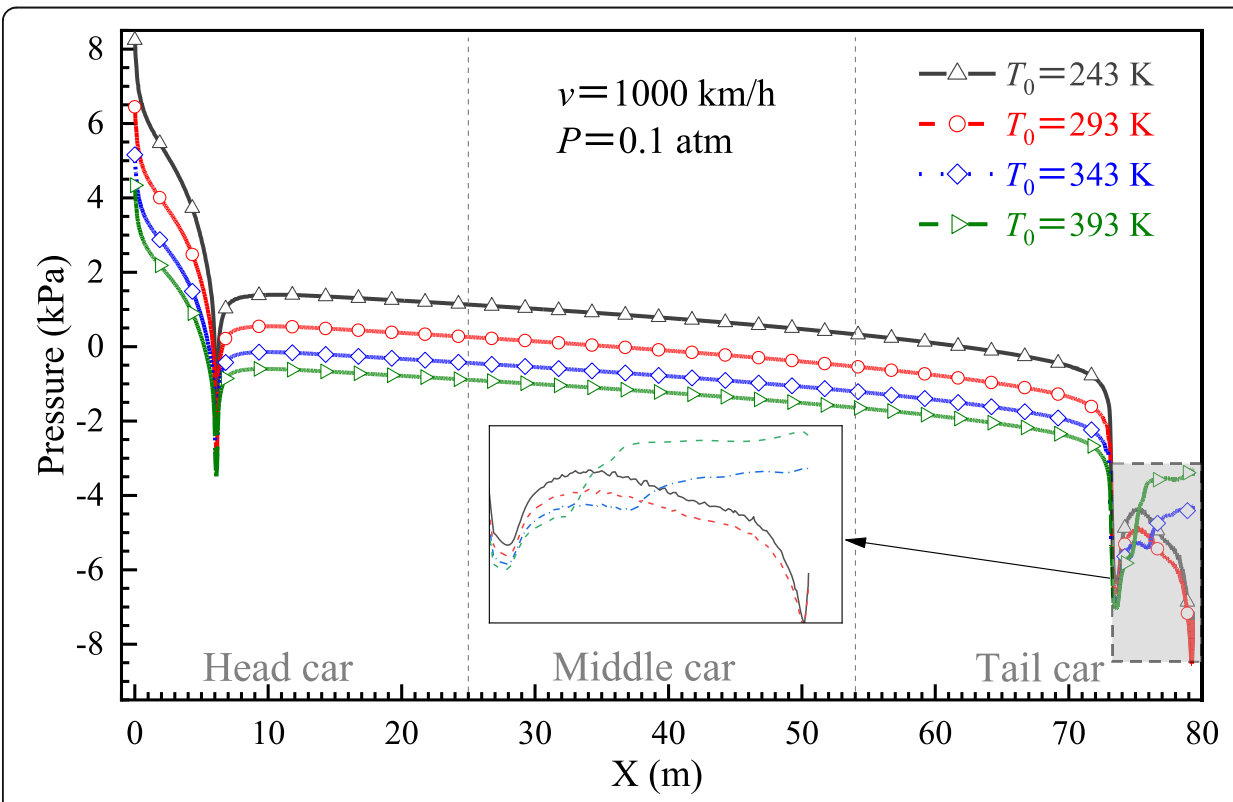

Fig. 13 Pressure distribution on the intersection line between the train upper surface and the $x y$ plane at different $T_{0}$ 


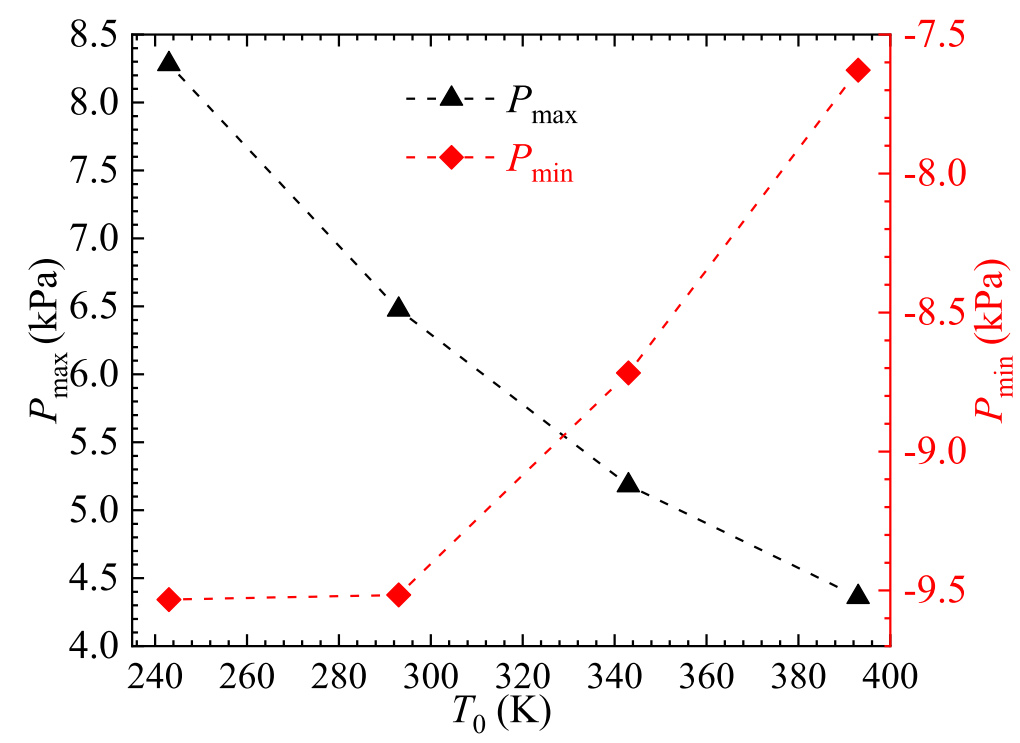

Fig. 14 Maximum and minimum pressure on the train surface at different $T_{0}$

shorter. Moreover, when $T_{0}$ rises from $243 \mathrm{~K}$ to $343 \mathrm{~K}$, the change of supersonic flow region is more obvious, while when $T_{0}$ rises from $343 \mathrm{~K}$ to $393 \mathrm{~K}$, the change of supersonic flow region is relatively small. Meanwhile, the maximum Mach number on this straight line also decreases with the increase of $T_{0}$. Especially, when $T_{0}$ rises from 293 $\mathrm{K}$ to $393 \mathrm{~K}$, the maximum Mach number changes greatly.

Subsequently, the pressure data was collected on the straight line which used in Fig. 16, and the pressure curves at different $T_{0}$ were plotted in Fig. 18. It can be found from Fig. 18 and Fig. 19 that $T_{0}$ also has a great influence on the air pressure. And with the rise of $T_{0}$, the air pressure gradually decreases. The influence of $T_{0}$ on the air pressure in the wake is similar to the influence of $T_{0}$ on the Mach number in the wake.

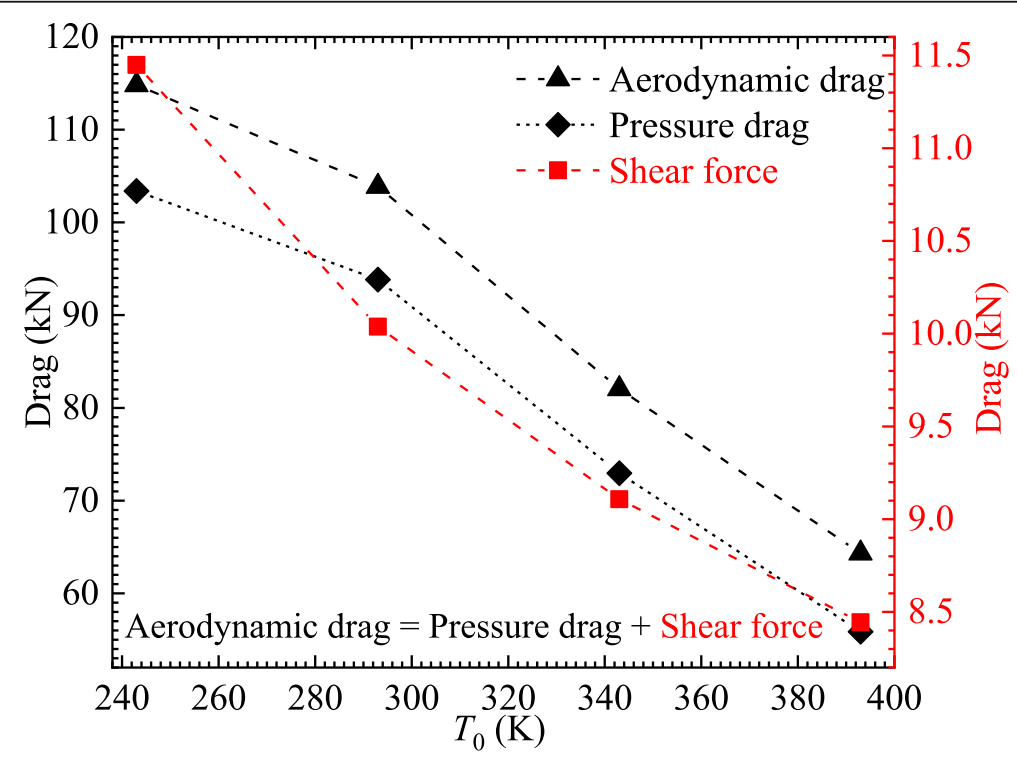

Fig. 15 Train aerodynamic drag at different $T_{0}$ 


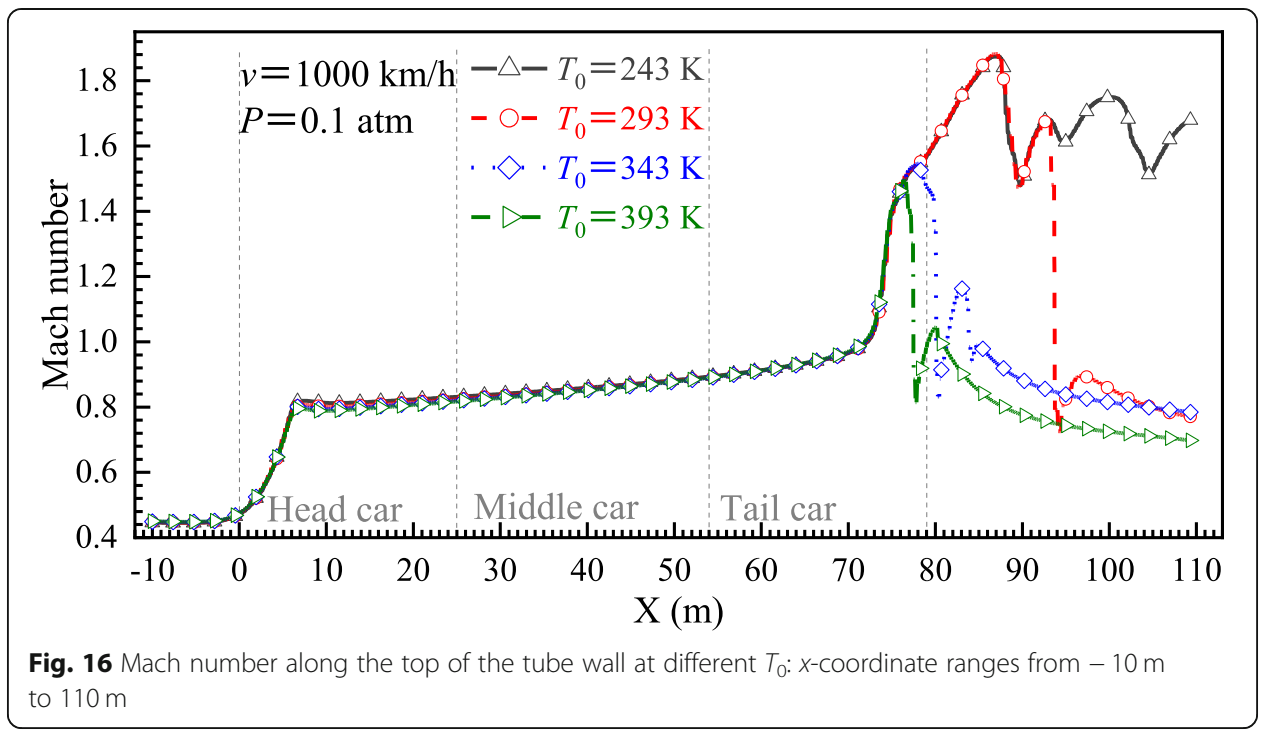

The reason for this phenomenon is probably due to the acceleration of the expansion of the airflow, resulting in faster airflow velocity and lower pressure, as analyzed in Section 4.1. Therefore, the air pressure will change with the change of the Mach number. Finally, according to the isentropic flow, the structure of the temperature field in the wake will also change with the Mach number and pressure.

As shown in Fig. 20, it can be seen from the contour of the temperature field in the wake area that $T_{0}$ has a great influence on the structure of temperature field. When the $T_{0}$ rises from $243 \mathrm{~K}$ to $293 \mathrm{~K}$, the length of the low temperature region in the wake area is shortened from about $98 \mathrm{~m}$ to about $21 \mathrm{~m}$, but the contours of their low temperature region have a similar profile. When $T_{0}$ continues to rise to $343 \mathrm{~K}$ and $393 \mathrm{~K}$, the length of its low temperature region is not only shortened, but also the profile of its temperature contour is changed.

\section{Conclusions}

This paper mainly studied the influence of initial ambient temperature $\left(T_{0}\right)$ on the aerodynamic heating at transonic speed $(1000 \mathrm{~km} / \mathrm{h})$, and also studied the temperature distribution in the tube. Some conclusions are drawn as follows:

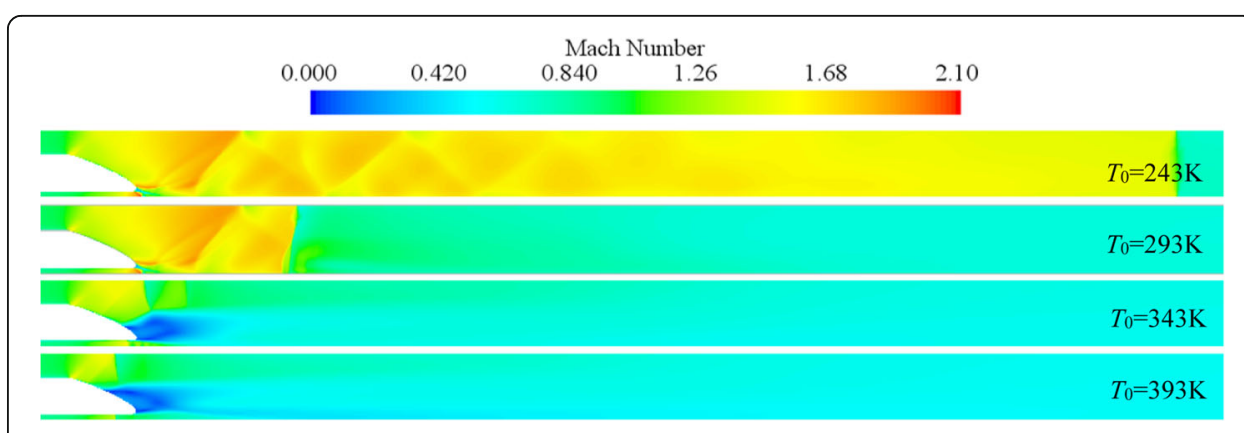

Fig. 17 The contours of Mach number in the wake area at different $T_{0}$ 


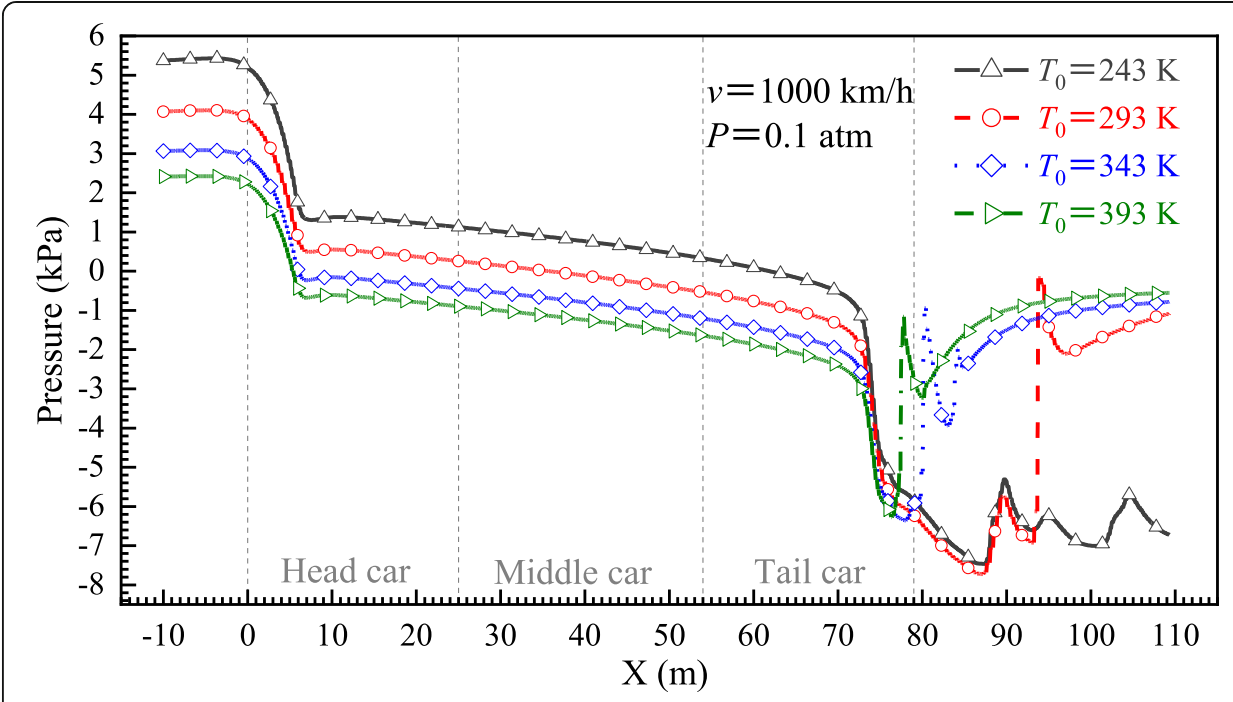

Fig. 18 Pressure along the top of the tube wall at different $T_{0}$ : $x$-coordinate ranges from $-10 \mathrm{~m}$ to $110 \mathrm{~m}$

(1) Because of the influence of aerodynamic heating, the temperature of the head car is higher than that of the tail car, and the maximum temperature of the train surface is located at the stagnation point of the nose tip of the head car. Due to the complex wake area, the temperature distribution of the tube wall in the wake area fluctuates greatly.

(2) When the train is running at transonic speed, the velocity of the air flow at the shoulder of the tail car reaches sonic speed and continues to accelerate, resulting in a supersonic flow area with low temperature and low-pressure in the wake area. At the same time, the structure of the wake flow field becomes complicated because of generation and disturbance of the shock wave.

(3) $T_{0}$ has a great influence on the aerodynamic drag of the train. When $T_{0}$ rises from $243 \mathrm{~K}$ to $393 \mathrm{~K}$, the aerodynamic drag of the train decreases by about $40 \%$.

(4) The increase of $T_{0}$ has a great influence on the structure of the flow field in the wake, which makes the length of the low temperature region shorter and the structure of the temperature distribution changes.

Considering the above, it is necessary to maintain the thermal equilibrium of the tube train system. If the heat dissipation of the tube is insufficient, it may lead to a higher

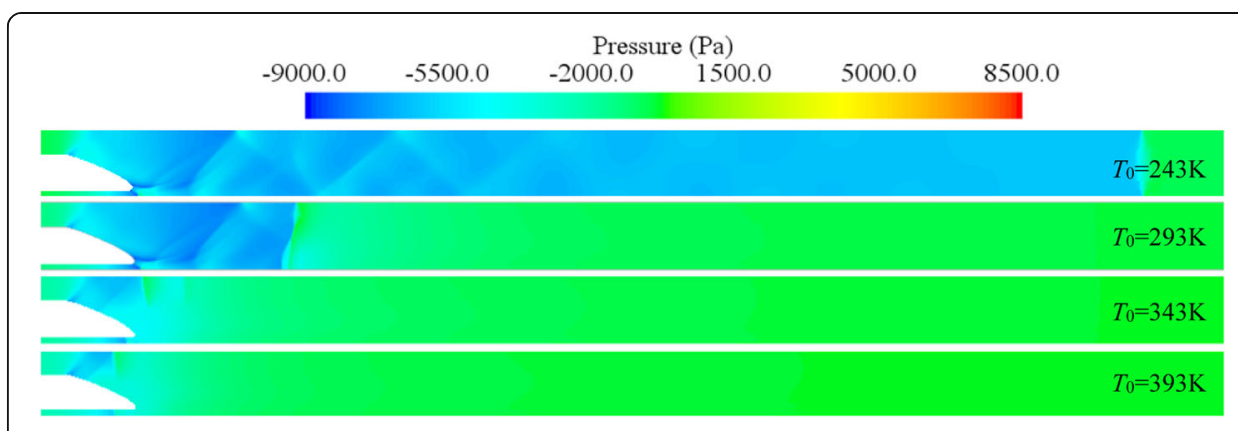

Fig. 19 The contours of pressure in the wake area at different $T_{0}$ 


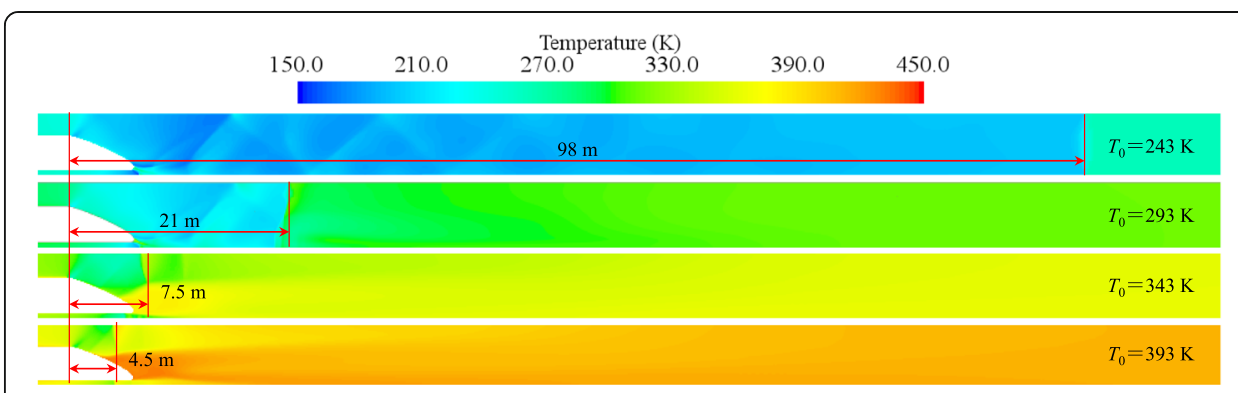

Fig. 20 The contours of temperature in the wake area at different $T_{0}$

and higher temperature rise and endanger the safety of the system. In addition, the low temperature region in the wake leads to a larger temperature difference, it may increase the temperature stress of the tube and affect the strength of the tube. And larger temperature change may also affect the normal operation of the equipment in the tube. Consequently, the temperature inside the tube can be maintained at a reasonable value to reduce the influence of the low temperature region in the wake on the system, as well as reduce the aerodynamic drag of the train. At present, the reasons for the influence of initial ambient temperature on the structure of the flow field are not very clear. Therefore, we will continue to study this problem and explore the influence mechanism.

\section{Acknowledgements}

The authors are grateful to Qiqi Zhang, Xue Song and Zhihao Ke for their help in improving the English writing of this article.

\section{Authors' contributions}

SB was a major contributor in writing the manuscript, and carried out the numerical simulation. $\mathrm{XH}$ directed the numerical simulation and put forward suggestions for the manuscript. JW and TM processed the calculated data. YR drew some diagrams. ZD reviewed and revised the manuscript. All authors read and approved the final manuscript.

\section{Funding}

This work was supported in part by the National Natural Science Foundation of China (U19A20102), the Science and Technology Partnership Program, Ministry of Science and Technology of China (KY201701001), the Sichuan Science and Technology Program (2019YJ0229), the Chengdu International S\&T Cooperation Program (2019-GH02-00002-HZ), the Fundamental Research Funds for the Central Universities (2682018CX72), and the State Key Laboratory of Traction Power at Southwest Jiaotong University (2019TPL_07).

\section{Availability of data and materials}

Not applicable.

\section{Competing interests}

The authors declare that they have no competing interests.

\section{Author details}

${ }^{1}$ School of Mechanics and Engineering, Southwest Jiaotong University, Chengdu 610031, China. ${ }^{2}$ State Key Laboratory of Traction Power, Southwest Jiaotong University, Chengdu 610031, China.

Received: 24 July 2020 Accepted: 10 November 2020

Published online: 08 December 2020

\section{References}

1. Deng ZG, Zhang Y, Wang B et al (2019) Present situation and prospect of evacuated tube transportation system. J Southwest Jiaotong Univ 54(5):1063-1072

2. Schetz JA (2001) Aerodynamics of high-speed trains. Annu Rev Fluid Mech 33(1):371-414

3. Shen ZY (2005) On developing high-speed evacuated tube transportation in China. J Southwest Jiaotong Univ 40(2):133-137

4 Shen ZY (2001) Dynamic interaction of high speed maglev train on girders and its comparison with the case in ordinary high speed railways. J Traffic Trans Eng 1(1):1-6

5. Talotte C (2000) Aerodynamic noise: a critical survey. J Sound Vib 231(3):549-562

6. Goddard HR (1945) Apparatus for vacuum tube transportation. US Patent 2488287 Oct. 1945

7. Oster D, Kumada M, Zhang YP (2011) Evacuated tube transport technologies (ET3) tm: a maximum value global transportation network for passengers and cargo. J Modern Transp 19(1):42-50

8. Zhang YP, Yu X (2006) On the safety of evacuated tube transportation. J Transpt Eng Inf 4(3):57-63 
9. Mossi M, Rossel P (2001) Swissmetro: a revolution in the high-speed passenger transport systems, 1st Swiss transport research conference, pp 1-16

10. Niu JQ, Sui Y, Yu QJ, Cao XL, Yuan YP (2019) Numerical study on the impact of Mach number on the coupling effect of aerodynamic heating and aerodynamic pressure caused by a tube train. J Wind Eng Ind Aerod 190:100-111

11. Zhou X, Zhang YP, Yao YF (2008) Numerical simulation on the aerodynamic drag of high-speed train in evacuated tube. Sci Technol Eng 8(6):1626-1628

12. Zhou X, Zhang D, Zhang YP (2008) Numerical simulation of blockage rate and aerodynamic drag of high-speed train in evacuated tube transportation. Chin J Vacuum Sci Technol 28(6):535-538

13. Mi BG, Zhan H, Zhu J (2013) Simulation of aerodynamic drag of high-speed train in evacuated tube transportation. Chin J Vacuum Sci Technol 33(9):877-882

14. Chen XY, Zhao LF, Ma JQ, Liu Y (2012) Aerodynamic simulation of evacuated tube maglev trains with different streamlined designs. J Modern Transp 20(2):115-120

15. Zhang YP (2012) Impact of tube section scale on the construction and operation costs of evacuated tube transportation. Vacuum 49(4):17-21

16. Ma JQ, Zhou DJ, Zhao LF, Zhang Y, Zhao YP (2013) The approach to calculate the aerodynamic drag of maglev train in the evacuated tube. J Modern Transp 21(3):200-208

17. Liu JL, Zhang JY, Zhang WH (2014) Impacts of pressure, blockage ratio and speed on aerodynamic drag force of highspeed trains. Chin J Vacuum Sci Technol 34(1):10-15

18. Bao SJ, Wang B, Zhang Y, Deng ZG (2017) Preliminary study of aerodynamic characteristics of high temperature superconducting maglev-evacuated tube transport system. 2nd International Conference on Industrial Aerodynamics (ICIA 2017), pp. 236-249. https://doi.org/10.12783/dtetr/icia2017/15646

19. Kim TK, Kim KH, Kwon HB (2011) Aerodynamic characteristics of a tube train. J Wind Eng Ind Aerod 99(12):1187-1196

20. Zhou P, Zhang JY, Li T, Zhang WH (2019) Numerical study on wave phenomena produced by the super high-speed evacuated tube maglev train. J Wind Eng Ind Aerod 190:100-111

21. Zhou P, Li T, Zhang JY, Zhang WH (2020) Research on shock wave trains generated by the hyper train in the evacuated tube. J Mechanical Eng 56(2):86-97

22. Liu JL, Zhang JY, Zhang WH (2013) Simulation of noise source for high speed train in evacuated tube. Chin J Vac Sci Technol 33(10):1026-1031

23. Zhang KR, Li QL, Wang CW, Jia WG (2019) Aerodynamic noises of vacuum tube transportation: a simulation and theoretical study. Chin J Vac Sci Technol 39(11):950-956

24. Jia WG, Dong CG, Zhou Y, Duan RX, Li QL (2013) Study of thermal-pressure coupling effect in the evacuated tube transportation system on blocking ratio. J Eng Thermophys 34(9):1745-1748

25. Duan RX (2014) Research on thermal-pressure coupling effect aerodynamic characteristics in the evacuated tube transport system. Dissertation, Qingdao University of Science and Technology, Qingdao

26. Dong CG (2013) The influence of blockage ratio on aerodynamic heating and heat transfer of the evacuated tube transportation system. Dissertation, Qingdao University of Science and Technology, Qingdao

27. Zhou P, Li T, Zhang JY, Zhang WH (2020) Aerothermal effect generated by hyper train in the evacuated tube. J Mechanical Eng 56(8):190-199

28. Sui Y et al (2020) An Aerothermal study of influence of blockage ratio on a supersonic tube train system. J Thermal Sci 1-12. Doi: https://doi.org/10.1007/s11630-020-1281-7

29. Deng ZG, Zhang WH, Zheng J, Wang B, Ren Y, Zheng XX, Zhang JH (2017) A high-temperature superconducting maglev-evacuated tube transport (HTS maglev-ETT) test system. IEEE Trans Appl Supercond 27(6):1-8

30. Pan S, Feng DH, Ding GH, Tian ZY, Yang YM, Li H (2010) Grid dependency and convergence of hypersonic Aerothermal simulation. Acta Aeronautica Et Astronautica Sinica 31(3):493-499

31. Menter FR (1994) Two-equation eddy-viscosity turbulence models for engineering applications. AIAA J 32(8):1598-1605

32. Muld TW, Efraimsson G, Henningson DS (2012) Flow structures around a high-speed train extracted using proper orthogonal decomposition and dynamic mode decomposition. Comput Fluids 57:87-97

33. Schmitt V, Charpin F (1979) Pressure Distributions on the ONERA-M6-Wing at Transonic Mach Numbers, Experimental Data Base for Computer Program Assessment. Report of the Fluid Dynamics Panel Working Group 04, AGARD AR 138

\section{Publisher's Note}

Springer Nature remains neutral with regard to jurisdictional claims in published maps and institutional affiliations.

\section{Submit your manuscript to a SpringerOpen ${ }^{\circ}$ journal and benefit from:}

- Convenient online submission

- Rigorous peer review

- Open access: articles freely available online

- High visibility within the field

- Retaining the copyright to your article

Submit your next manuscript at $\boldsymbol{\nabla}$ springeropen.com 